\title{
UNIFORMLY CONVEX FUNCTIONS AND A CORRESPONDING CLASS OF STARLIKE FUNCTIONS
}

\author{
FRODE RØNNING
}

(Communicated by Clifford J. Earle, Jr.)

\begin{abstract}
We investigate starlike functions $f(z)=z+\sum_{k=2}^{\infty} a_{k} z^{k}$ with the property that $z f^{\prime}(z) / f(z)$ lies inside a certain parabola. These functions are closely related to a class of functions called uniformly convex and recently introduced by Goodman. We give some particular examples of functions having the required properties, and we give upper bounds on the coefficients and the modulus $|f(z)|$ of the functions in the class.
\end{abstract}

\section{INTRODUCTION}

We shall be concerned with functions of the form

$$
f(z)=z+\sum_{k=2}^{\infty} a_{k} z^{k}
$$

analytic and univalent in the unit disk $U$. Goodman $[4,5]$ introduced the concepts of uniform convexity and uniform starlikeness for such functions, thereby providing proper subclasses of the usual classes of convex and starlike functions, here denoted by $K_{0}$ and $S_{0}$, respectively. The corresponding "uniform classes" are defined in the following way, by their geometrical mapping properties.

Definition 1. A function $f$ is said to be uniformly convex (starlike) in $U$ if $f$ is in $K_{0}\left(S_{0}\right)$ and has the property that for every circular arc $\gamma$ contained in $U$, with center $\zeta$, also in $U$, the arc $f(\gamma)$ is convex (starlike w.r.t. $f(\zeta)$ ).

Following Goodman's notation, we denote these classes by UCV (UST). That this definition really gives proper subclasses of $K_{0}$ and $S_{0}$ is established through numerous results in $[4,5]$. An analytic description of $U C V$ and UST can be found in [5]. We state this as

Theorem A. Let $f(z)=z+\sum_{k=2}^{\infty} a_{k} z^{k}$. Then

(a) $f \in U C V$ if and only if

$$
\operatorname{Re}\left\{1+(z-\zeta) \frac{f^{\prime \prime}(z)}{f^{\prime}(z)}\right\} \geq 0, \quad(z, \zeta) \in U \times U .
$$

Received by the editors May 13, 1991 and, in revised form, September 11, 1991. 1991 Mathematics Subject Classification. Primary 30C45. 
(b) $f \in U S T$ if and only if

$$
\operatorname{Re} \frac{f(z)-f(\zeta)}{(z-\zeta) f^{\prime}(z)} \geq 0, \quad(z, \zeta) \in U \times U
$$

Note that by taking $\zeta=0$ in (1.2) and (1.3) we are back to the classes $K_{0}$ and $S_{0}$. The classic Alexander's theorem stating that $f \in K_{0} \Leftrightarrow z f^{\prime}(z) \in S_{0}$ provides a bridge between these two classes. One might hope that there would be a similar bridge between $U C V$ and $U S T$, but two examples in [4] show that this is not the case. However, there is still a hope that there might be a "one way bridge" in the sense that $f \in U C V$ implies $z f^{\prime}(z) \in U S T$. In this paper we proceed in another way, by building the bridge and exploring where it leads, i.e., we define a class of starlike functions $S_{p}$ in the following way.

Definition 2. $S_{p}:=\left\{F \in S_{0} \mid F(z)=z f^{\prime}(z), f \in U C V\right\}$.

In what follows, we shall investigate the class $S_{p}$, and the results that we get can be directly translated to results about $U C V$. The question of the "one way bridge", which could be expressed as $S_{p} \subset U S T$, is still an open problem. In the present paper we give a simple example (Theorem 2) supporting the conjecture $S_{p} \subset U S T$.

\section{A ONE VARIABLE CHARACTERIZATION OF $U C V$}

We start by stating the theorem that is the basis for our further investigations of $S_{p}$ and $U C V$.

Theorem 1. Let $g(z)=z+\sum_{k=2}^{\infty} a_{k} z^{k}$. Then $g \in U C V$ if and only if

$$
\operatorname{Re}\left\{1+\frac{z g^{\prime \prime}(z)}{g^{\prime}(z)}\right\}>\left|\frac{z g^{\prime \prime}(z)}{g^{\prime}(z)}\right|, \quad z \in U .
$$

Proof. Let $g \in U C V$. Then from (1.2), we have equivalently

$$
\operatorname{Re}\left\{1+\frac{z g^{\prime \prime}(z)}{g^{\prime}(z)}\right\} \geq \operatorname{Re} \frac{\zeta g^{\prime \prime}(z)}{g^{\prime}(z)}
$$

for every $z$ and $\zeta$ in $U$. Choosing $\zeta=e^{i \alpha} z$ in a suitable way we will get $\operatorname{Re} \zeta g^{\prime \prime}(z) / g^{\prime}(z)=\left|z g^{\prime \prime}(z) / g^{\prime}(z)\right|$. Hence, a necessary condition for $g \in U C V$ is that

$$
\operatorname{Re}\left\{1+\frac{z g^{\prime \prime}(z)}{g^{\prime}(z)}\right\} \geq\left|\frac{z g^{\prime \prime}(z)}{g^{\prime}(z)}\right|, \quad z \in U .
$$

We show that (2.3) is also a sufficient condition. Let $\zeta \in U$ be an arbitrary, but from now on, fixed point in $U . \operatorname{Re}\left\{1+(z-\zeta) g^{\prime \prime}(z) / g^{\prime}(z)\right\}$ is a harmonic function, so by the minimum principle it is enough to show (2.2) for $|z|=R>$ $|\zeta|, R<1$. Assume (2.3) holds. Then for $1>|z|=R>|\zeta|$, we have

$$
\operatorname{Re}\left\{1+\frac{z g^{\prime \prime}(z)}{g^{\prime}(z)}\right\} \geq\left|\frac{z g^{\prime \prime}(z)}{g^{\prime}(z)}\right|>\left|\frac{\zeta g^{\prime \prime}(z)}{g^{\prime}(z)}\right| \geq \operatorname{Re} \frac{\zeta g^{\prime \prime}(z)}{g^{\prime}(z)},
$$

which is (2.2). Hence (2.2) and (2.3) are equivalent, and since $1+z g^{\prime \prime}(z) / g^{\prime}(z)$ is analytic in $U$ and maps 0 to 1 , the Open Mapping Theorem implies that equality in (2.3) is not possible.

Taking $f(z)=z g^{\prime}(z)$, we immediately get 
Corollary 1. A function $f(z)$, with $f(0)=f^{\prime}(0)-1=0$, is in $S_{p}$ if and only if

$$
\left|\frac{z f^{\prime}(z)}{f(z)}-1\right|<\operatorname{Re} \frac{z f^{\prime}(z)}{f(z)}, \quad z \in U .
$$

Interpreting (2.4) geometrically, we see that $S_{p}$ is the class of functions for which the domain of values of $z f^{\prime}(z) / f(z), z \in U$, is the parabolic region $(\operatorname{Im} w)^{2}<2 \operatorname{Re} w-1$. In particular, we get for $f \in S_{p}$ that

$$
\operatorname{Re} \frac{z f^{\prime}(z)}{f(z)}>\frac{1}{2}
$$

and

$$
\left|\arg \frac{z f^{\prime}(z)}{f(z)}\right|<\frac{\pi}{4}
$$

Both conditions (2.5) and (2.6) define well-known subclasses of $S_{0}$. The inequality (2.5) defines the class of functions starlike of order $\frac{1}{2}$, here denoted by $S_{1 / 2}$. For the functions satisfying $(2.6)$, we refer to the papers [2,3], which contain several results about these functions called functions strongly starlike of order $\frac{1}{2}$. We denote this class by $S S_{1 / 2}$.

Both these and other interesting subclasses of $S_{0}$ are characterized by the domain of values $\Omega$ of the functional $z f^{\prime}(z) / f(z)$. To know a function $P(z)$, analytic and univalent in $U$, which maps $U$ onto $\Omega$, is helpful for investigating the class of functions in question. In the case $S_{p}, \Omega$ is the parabolic region $|w-1|<\operatorname{Re} w$, and the appropriate mapping $P: U \rightarrow \Omega$ with the normalization $P(0)=1$ is seen to be

$$
P(z)=1+\frac{2}{\pi^{2}}\left(\log \frac{1+\sqrt{z}}{1-\sqrt{z}}\right)^{2} .
$$

(The branch of $\sqrt{z}$ is chosen such that $\operatorname{Im} \sqrt{z} \geq 0$.)

Using the expansion $\log (1+\sqrt{z}) /(1-\sqrt{z})=2 \sqrt{z} \sum_{k=0}^{\infty} z^{k} /(2 k+1)$, we get the power series expansion for $P(z)$,

$$
\begin{aligned}
P(z) & =1+\frac{8}{\pi^{2}} \sum_{k=1}^{\infty}\left(\sum_{m=1}^{k} \frac{1}{4 m-4 m^{2}+4 m k-1-2 k}\right) z^{k} \\
& =1+\frac{8}{\pi^{2}}\left(z+\frac{2}{3} z^{2}+\frac{23}{45} z^{3}+\frac{44}{105} z^{4}+\cdots\right) .
\end{aligned}
$$

\section{SOME SPECiAl MEMBers of $S_{p}$}

Theorem 2. $f(z)=z+a_{n} z^{n}$ is in $S_{p}$ if and only if $\left|a_{n}\right| \leq 1 /(2 n-1)$.

Proof. It suffices to study (2.4) for $|z|=1$. Set $\left|a_{n}\right|=r$ and $a_{n} z^{n-1}=r e^{i \varphi}$. Then (2.4) for this $f$ will be

$$
\left|\frac{(n-1) r e^{i \varphi}}{1+r e^{i \varphi}}\right| \leq \operatorname{Re} \frac{1+n r e^{i \varphi}}{1+r e^{i \varphi}} .
$$

By computing we see that

$$
\operatorname{Re} \frac{1+n r e^{i \varphi}}{1+r e^{i \varphi}}=\frac{1+n r^{2}+(n+1) r \cos \varphi}{\left|1+r e^{i \varphi}\right|^{2}},
$$


so (3.1) is equivalent to

$$
(n-1) r \leq \frac{1+n r^{2}+(n+1) r \cos \varphi}{\left|1+r e^{i \varphi}\right|}=\frac{1+n r^{2}+(n+1) r \cos \varphi}{\left(1+r^{2}+2 r \cos \varphi\right)^{1 / 2}} .
$$

The right-hand side of (3.2) is seen to have a minimum for $\varphi=\pi$, and this minimal value is $1-n r$. Hence, a necessary and sufficient condition for (3.2) is $(n-1) r \leq 1-n r$ or $\left|a_{n}\right|=r \leq 1 /(2 n-1)$.

Goodman [5] has proved that $\left|a_{n}\right| \leq \sqrt{2} / 2 n$ is sufficient for $f(z)=z+$ $a_{n} z^{n}$ to be in UST. Note that Theorem 2 is more restrictive than this, so the inclusion $S_{p} \subset U S T$ is possible.

Corollary 2. $g(z)=z+b_{n} z^{n}$ is in $U C V$ if and only if $\left|b_{n}\right| \leq 1 / n(2 n-1)$.

For the case $n=2$ this has also been proved in [4, Lemma 2].

A natural question would be to ask for how large $z$ the Koebe function $k(z)=z /(1-z)^{2}$ will have the properties of $S_{p}$. This question has indirectly been answered by Goodman [4, Theorem 3] by looking at the corresponding function $z /(1-z)=\int_{0}^{z} k(\zeta) / \zeta d \zeta$ in $U C V$. We state the result here for completeness.

Theorem 3. $k(z)=z /(1-A z)^{2}$ is in $S_{p}$ if and only if $|A| \leq \frac{1}{3}$.

It is easily seen, and well known, that the range of values of the functional $z f^{\prime}(z) / f(z)$ for $f \in S_{0}$ is determined by the Koebe function alone. Hence, we immediately get what we could call the $S_{p}$ radius in $S_{0}$.

Corollary 3. If $f \in S_{0}$, then $\frac{1}{r} f(r z) \in S_{p}$ if and only if $r \leq \frac{1}{3}$.

Furthermore, from the properties of the Koebe function, it is clear that if $f$ is restricted to $S_{0}$, then $F(z):=f(r z) / r$ is in $S_{p}$ if and only if $\operatorname{Re} z f^{\prime}(z) / f(z) \geq$ $\frac{1}{2}$. Hence the $S_{p}$ radius in $S_{0}$ is equal to the $S_{1 / 2}$ radius. If we turn to the whole class $S$ of normalized univalent functions then the $S_{p}$ radius and the $S_{1 / 2}$ radius will not coincide, but as the next theorem shows, the difference between the two radii is very small.

Theorem 4. If $f \in S$, then the function $\frac{1}{r} f(r z)$ is in $S_{p}$ if and only if $r \leq$ $0.33217 \ldots$ and in $S_{1 / 2}$ if and only if $r \leq \frac{1}{3}$.

Proof. For $f \in S$ we have the following (sharp) inequality (see, e.g., [6, p. 168]):

$$
\left|\log \frac{z f^{\prime}(z)}{f(z)}\right| \leq \log \frac{1+|z|}{1-|z|}, \quad z \in U .
$$

Using this, the boundary of the range of $z f^{\prime}(z) / f(z), f \in S,|z|=r$, can be parameterized

$$
\begin{aligned}
W_{r}(\theta)=\left(\frac{1+r}{1-r}\right)^{\cos \theta}( & \cos \left(\sin \theta \log \frac{1+r}{1-r}\right) \\
& \left.+i \sin \left(\sin \theta \log \frac{1+r}{1-r}\right)\right), \quad \theta \in[0,2 \pi\rangle .
\end{aligned}
$$

We now search for the largest $r$ for which

$$
\left|W_{r}(\theta)-1\right| \leq \operatorname{Re} W_{r}(\theta), \quad \theta \in[0,2 \pi\rangle .
$$


$W_{r}(\theta)$ is a smooth, simple, closed curve, symmetric about the real axis. A straightforward calculation shows that

$$
\tan \left(\arg \left\{\frac{\partial}{\partial \theta} W_{r}(\theta)\right\}\right)=-\frac{1}{\tan (\theta+\sin \theta \log (1+r) /(1-r))},
$$

hence

$$
\arg \left\{\frac{\partial}{\partial \theta} W_{r}(\theta)\right\}=\theta+\sin \theta \log \frac{1+r}{1-r}+\frac{\pi}{2}
$$

and

$$
\frac{\partial}{\partial \theta}\left(\arg \left\{\frac{\partial}{\partial \theta} W_{r}(\theta)\right\}\right)=1+\cos \theta \log \frac{1+r}{1-r} \geq 1-\log \frac{1+r}{1-r}>0
$$

for $r<(e-1) /(e+1) \approx 0.462 \ldots$, which shows that $W_{r}(\theta)$ is even convex for those values of $r\left(\leq \frac{1}{3}\right)$ that are of interest here. If we denote by $\Omega_{r}$ the domain enclosed by $W_{r}(\theta)$ then $1 \in \Omega_{r}$ for all $r \in[0,1\rangle$, and if $r_{1}<r_{2}$ then $\Omega_{r_{1}} \subset \Omega_{r_{2}}$. These properties ensure that the value of $r$ that we are looking for is the smallest (positive) value of $r$ that is a solution of the equation system

$$
\begin{gathered}
\left(\frac{1+r}{1-r}\right)^{\cos \theta} \cos \left(\sin \theta \log \frac{1+r}{1-r}\right) \\
=\frac{1}{2}\left(\left(\frac{1+r}{1-r}\right)^{2 \cos \theta} \sin ^{2}\left(\sin \theta \log \frac{1+r}{1-r}\right)+1\right) \\
-\tan \left(\theta+\sin \theta \log \frac{1+r}{1-r}\right)=\left(\frac{1+r}{1-r}\right)^{\cos \theta} \sin \left(\sin \theta \log \frac{1+r}{1-r}\right) .
\end{gathered}
$$

Here (3.4) states that $W_{r}(\theta)$ intersects the parabola, and (3.5) states that $W_{r}(\theta)$ and the parabola have parallel tangents. We see that one solution of the system is $(r, \theta)=\left(\frac{1}{3}, \pi\right)$, which geometrically means that $W_{r}(\theta)$ is tangent to the parabola "from the outside". To find nontrivial solutions we have used the computer system MAPLE working with 15 digits precision. This provided the solution $r=0.3321758 \ldots$ and $\theta=\pi \pm 0.6240519 \ldots$. From the geometrical properties of the two curves we conclude that this value of $r$ is the one that we want.

From (3.3) we see that $W_{r}(\theta)$ has a vertical tangent for $\theta=0$, and since $W_{r}(\theta)$ is convex, it is clear that

$$
\operatorname{Re} \frac{z f^{\prime}(z)}{f(z)} \geq \frac{1-|z|}{1+|z|}>\frac{1}{2} \text { for }|z|<\frac{1}{3},
$$

so the $S_{1 / 2}$ radius in $S$ is $\frac{1}{3}$, i.e., slightly larger than the $S_{p}$ radius.

\section{General properties of fUNCTIONS IN $S_{p}$}

The question of bounds for the coefficients is a classical one in the theory of univalent functions. For functions in $S_{1 / 2}$ it has long been known [8] that $\left|a_{n}\right| \leq 1$ (sharp bound), and since $S_{p} \subset S_{1 / 2}$, the same bound must hold in $S_{p}$, but not necessarily as a sharp one. As we have mentioned before, $S_{p} \subset$ 
$S_{1 / 2} \cap S S_{1 / 2}$ (see (2.5) and (2.6)), so the coefficient bounds for $S S_{1 / 2}$ must also hold in $S_{p}$. For $S S_{1 / 2}$ we find in [3] the bounds $\left|a_{2}\right| \leq 1$ and $\left|a_{3}\right| \leq \frac{3}{4}$ (both sharp). A bound for $\left|a_{n}\right|, n>3$, in $S S_{1 / 2}$ is (as far as we know) not known. In the class $S_{p}$, however, we are able to get bounds on $\left|a_{n}\right|$, but they are not sharp, except in the case $n=2$. However, they are lower than the corresponding bounds in $S_{1 / 2}$ and $S S_{1 / 2}$, so they represent nontrivial results.

Theorem 5. Let $f(z)=z+\sum_{k=2}^{\infty} a_{k} z^{k}$, and let $A_{n}=\max _{f \in S_{p}}\left|a_{n}\right|$. Then

$$
\begin{aligned}
& A_{2}=\frac{8}{\pi^{2}}(\approx 0.81), \\
& A_{n} \leq \frac{8}{(n-1) \pi^{2}} \prod_{k=3}^{n}\left(1+\frac{8}{(k-2) \pi^{2}}\right), \quad n \geq 3 .
\end{aligned}
$$

For the proof of this theorem we need the following result by Rogosinski [7].

Rogosinski's theorem. Let $h(z)=1+\sum_{k=1}^{\infty} c_{k} z^{k}$ be subordinate to $H(z)=$ $1+\sum_{k=1}^{\infty} C_{k} z^{k}$ in $U$. If $H(z)$ is univalent in $U$ and $H(U)$ is convex, then $\left|c_{n}\right| \leq\left|C_{1}\right|$.

Proof of Theorem 5. Let $f(z)=z+\sum_{k=2}^{\infty} a_{k} z^{k} \in S_{p}$, and define $\varphi(z)=$ $z f^{\prime}(z) / f(z)=1+\sum_{k=1}^{\infty} c_{k} z^{k}$. Then $\varphi \prec P(\prec$ denotes subordination), where $P$ is the function in (2.7). $P(z)$ is univalent in $U$ and $P(U)$, the parabola $|w-1|<\operatorname{Re} w$, is a convex region, so Rogosinski's theorem applies. $P(z)=$ $1+\left(8 / \pi^{2}\right) z+\cdots$, so we have $\left|c_{n}\right| \leq 8 / \pi^{2}:=c$. Now, writing $z f^{\prime}(z)=\varphi(z) f(z)$ and comparing coefficients of $z^{n}$ on both sides, we get

$$
(n-1) a_{n}=\sum_{k=1}^{n-1} c_{n-k} a_{k} .
$$

From this we get $\left|a_{2}\right|=\left|c_{1}\right| \leq 8 / \pi^{2}$. If we choose $f$ to be that function for which $z f^{\prime}(z) / f(z)=P(z)$, then $f$ is a function in $S_{p}$ with $a_{2}=8 / \pi^{2}$, which shows that this result is sharp. Further we get

$$
\left|a_{3}\right| \leq \frac{1}{2}\left|c_{2}+c_{1} a_{2}\right| \leq \frac{1}{2}\left(\left|c_{2}\right|+\left|c_{1}\right|\left|a_{2}\right|\right) \leq \frac{1}{2} c(1+c) \approx 0.73 .
$$

We now proceed by induction. Assume we have

$$
\left|a_{k}\right| \leq \frac{c}{k-1}(1+c)\left(1+\frac{c}{2}\right) \cdots\left(1+\frac{c}{k-2}\right), \quad \text { for } k=3,4, \ldots, n-1 .
$$

Then

$$
\begin{aligned}
(n-1)\left|a_{n}\right| \leq & \sum_{k=1}^{n-1}\left|c_{n-k}\right|\left|a_{k}\right| \leq c \sum_{k=1}^{n-1}\left|a_{k}\right| \\
\leq & c\left(1+c+\frac{c}{2}(1+c)+\frac{c}{3}(1+c)\left(1+\frac{c}{2}\right)\right. \\
& \left.\quad+\cdots+\frac{c}{n-2}(1+c)\left(1+\frac{c}{2}\right) \cdots\left(1+\frac{c}{n-3}\right)\right) \\
= & c(1+c)\left(1+\frac{c}{2}\right) \cdots\left(1+\frac{c}{n-2}\right)
\end{aligned}
$$


and

$$
\left|a_{n}\right| \leq \frac{c}{n-1} \prod_{k=3}^{n}\left(1+\frac{c}{k-2}\right) .
$$

For the class $S S_{1 / 2}$ there is a fixed number $M$ such that $|f(z) / z| \leq M$, $z \in U$. In [2] it is proved that $M=\frac{1}{4} \exp \left(-\Gamma^{\prime}\left(\frac{1}{4}\right) / \Gamma\left(\frac{1}{4}\right)-\gamma\right) \approx 9.62$, where $\Gamma(z)$ is the Gamma function and $\gamma$ is Euler's constant. Using the idea from the proof of this result in [2] we are able to get a corresponding result for $f \in S_{p}$ with a value of the upper bound that is considerably smaller.

Theorem 6. If $f \in S_{p}$, then

$$
|f(z)| \leq|z| \exp \left(\frac{14}{\pi^{2}} \zeta(3)\right)=|z| K
$$

for $|z|<1$. There is a function $f(z)$ in $S_{p}$ for which $\sup _{z \in U}|f(z)|=K \approx$ 5.502 .

$(\zeta(t)$ is the Riemann Zeta function.)

Proof. Let $\varphi(z)=z f^{\prime}(z) / f(z)$. Then

$$
\varphi(z) \prec 1+\frac{2}{\pi^{2}}\left(\log \frac{1+\sqrt{z}}{1-\sqrt{z}}\right)^{2} .
$$

Moreover,

$$
\log \frac{f(z)}{z}=\int_{0}^{z}(\varphi(\xi)-1) \frac{d \xi}{\xi}
$$

and therefore, if $z=r e^{i \theta}$,

$$
\begin{aligned}
\log \left|\frac{f(z)}{z}\right| & =\int_{0}^{r} \operatorname{Re}\left(\varphi\left(t e^{i \theta}\right)-1\right) \frac{d t}{t} \leq \frac{2}{\pi^{2}} \int_{0}^{r} \frac{1}{t}\left(\log \frac{1+\sqrt{t}}{1-\sqrt{t}}\right)^{2} d t \\
& \leq \frac{2}{\pi^{2}} \int_{0}^{1} \frac{1}{t}\left(\log \frac{1+\sqrt{t}}{1-\sqrt{t}}\right)^{2} d t=\frac{2}{\pi^{2}} \mathscr{I}_{1} .
\end{aligned}
$$

Substituting $u=\sqrt{t}$ and $v=\log (1+u) /(1-u)$ in $\mathscr{F}_{1}$, we get

$$
\begin{aligned}
\mathscr{I}_{1} & =\int_{0}^{1} \frac{1}{t}\left(\log \frac{1+\sqrt{t}}{1-\sqrt{t}}\right)^{2} d t \\
& =4 \int_{0}^{\infty} \frac{v^{2} e^{v}}{e^{2 v}-1} d v=\frac{1}{2} \int_{0}^{\infty} \frac{v^{2} e^{-v / 2}}{1-e^{-v}} d v=\frac{1}{2} \mathscr{J}_{2} .
\end{aligned}
$$

Using a formula for the generalized Zeta function in [9, pp. 265-266] we get

$$
\mathscr{I}_{2}=\Gamma(3) \sum_{n=0}^{\infty} \frac{1}{(n+1 / 2)^{3}}=16 \sum_{n=0}^{\infty} \frac{1}{(2 n+1)^{3}}
$$

$(\Gamma(3)=2)$. The series $\sum_{n=0}^{\infty} 1 /(2 n+1)^{3}$ is equal to $\frac{7}{8} \zeta(3)[1, \mathrm{p} .807]$, so we get $\mathscr{I}_{1}=\frac{1}{2} \mathscr{I}_{2}=7 \zeta(3)$, and (4.1) follows.

The equation

$$
\frac{z f^{\prime}(z)}{f(z)}=1+\frac{2}{\pi^{2}}\left(\log \frac{1+\sqrt{z}}{1-\sqrt{z}}\right)^{2}
$$

defines a function $f \in S_{p}$ for which $\sup _{z \in U}|f(z)|=|z| K$. 
It is clear how both Theorems 5 and 6 give results about the class $U C V$ by the correspondence $g \in U C V \Leftrightarrow z g^{\prime}(z) \in S_{p}$. Note that Theorem 5 improves results about bounds for the coefficients in $U C V$ proved in [4].

\section{REFERENCES}

1. M. Abramowitz and I. A. Stegun, Handbook of mathematical functions, Dover, New York, 1970.

2. D. A. Brannan and W. E. Kirwan, On some classes of bounded univalent functions, J. London Math. Soc.(2) 1 (1969), 431-443.

3. D. A. Brannan, J. Clunie, and W. E. Kirwan, Coefficient estimates for a class of starlike functions, Canad. J. Math. 22 (1970), 476-485.

4. A. W. Goodman, On uniformly convex functions, preprint.

5. __ On uniformly starlike functions, J. Math. Anal. Appl. 155 (1991), 364-370.

6. Chr. Pommerenke, Univalent functions, Vandenhoeck \& Ruprect, Göttingen, 1975.

7. W. Rogosinski, On the coefficients of subordinate functions, Proc. London Math. Soc. 48 (1943), 48-82.

8. A. Schild, On a class of univalent, star shaped mappings, Proc. Amer. Math. Soc. 9 (1958), 751-757.

9. E. T. Whittaker and G. N. Watson, A course of modern analysis, 4th ed., Cambridge Univ. Press, Cambridge, 1927.

Trondheim College of Education, Rotvoll Allé, N-7050 Charlottenlund, Norway E-mail address: frode.ronning@avh.unit.no 